\title{
Short-term learning of a visually guided power-grip task is associated with dynamic changes in EEG oscillatory activity
}

Kranczioch, C., Kranczioch, C., Athanassiou, S., Shen, S., Gao, G., Sterr, A., Gao, G.

\begin{abstract}
Objective

Performing a motor task after a period of training has been associated with reduced cortical activity and changes in oscillatory brain activity. Little is known about whether learning also affects the neural network associated with motor preparation and post movement processes. Here we investigate how short-term motor learning affects oscillatory brain activity during the preparation, execution, and postmovement stage of a force-feedback task.
\end{abstract}

\section{Methods}

Participants performed a visually guided power-grip tracking task. EEG was recorded from 64 scalp electrodes. Power and coherence data for the early and late stages of the task were compared.

Results

Performance improved with practice. During the preparation for the task alpha power was reduced for late experimental blocks. A movement execution-related decrease in beta power was attenuated with increasing task practice. A post-movement increase in alpha and lower beta activity was observed that decreased with learning. Coherence analysis revealed changes in cortico-cortical coupling with regard to the stage of the visuomotor task and with regard to learning. Learning was variably associated with increased coherence between contralateral and/or ipsilateral frontal and parietal, fronto-central, and occipital brain regions.

\section{Conclusions}

Practice of a visuomotor power-grip task is associated with various changes in the activity of a widespread cortical network. These changes might promote visuomotor learning.

\section{Significance}

This study provides important new evidence for and sheds new light on the complex nature of the brain processes underlying visuomotor integration and short-term learning.

\section{Introduction}

The ability to continuously adapt motor output based on visual feedback requires the integration of activity in a network of frontal, parietal and sensorimotor brain regions ( [Floyer-Lea and Matthews, 2004] and [Vaillancourt et al., 2003]). The electrophysiological signature of visuomotor integration is a decrease in oscillatory activity, in particular in the alpha and lower beta $(8-21 \mathrm{~Hz})$ frequency bands ( [Classen et al., 1998] and [Rearick et al., 2001]). Another variable to which visuomotor integration has been related to is long-range oscillatory neuronal synchronisation in the beta and gamma frequency ranges (13-80 Hz) ( [Aoki et al., 1999], [Babiloni et al., 2006], [Baker et al., 1999], [Lee, 2003] and [Ohara et al., 2000]). In particular, the finding of high-frequency synchronisation between visual, parietal, and motor cortices ( [Classen et al., 1998] and [Roelfsema et al., 1997]) suggests that the synchronisation or coherence of neuronal activity across distant brain regions might be the neural mechanism by which visuomotor integration is implemented. 
Oscillatory activity and coherence may not only reflect integration per se, but also subserve visuomotor learning, that is, an increase in the automaticity by which sensory information and motor parameters are integrated. This is suggested by recent research associating performance improvements in bimanual coordination tasks with an increase in long-range synchronisation between prefrontal areas, but also decreased synchronisation between primary sensorimotor regions and the midline area ( [Andres et al., 1999] and [Serrien and Brown, 2003]). Smith et al. (1999) report a taskspecific enhancement in alpha activity over somatomotor areas following several days of practicing a visuomotor tracking task, reflecting a decrease in cortical activation after practice. They propose that regional changes in alpha activity might reflect the development of task-specific neurocognitive strategies. It has also been shown on various occasions that repetition of a visuomotor task leads to improved performance and increased automaticity, and is accompanied by changes in brain activity as reflected in the event-related potential (ERP) and blood-oxygen level-dependent functional magnetic resonance imaging (BOLD fMRI) ( [Floyer-Lea and Matthews, 2004], [Halder et al., 2005] and [Staines et al., 2002]). A potential role of oscillatory brain activity and coherence in learning is also illustrated by a study on associative visuotactile learning (Miltner et al., 1999) that showed an increase of coherence with learning between brain regions involved in the associative learning task.

Aim of the present study was to investigate whether activity within the network active during the different stages of a visuomotor integration or motor adaptation (Doyon and Benali, 2005) task is affected by learning. Learning was defined as an improvement in task performance (Ungerleider et al., 2002), that is, a reduction in errors, in the late phase as compared to the early phase of the experiment. This definition of learning is compatible with the notion of short-term learning, that is, the early, fast learning stage of a motor skill in which considerable improvement in performance can be seen within a single training session ( [Floyer-Lea and Matthews, 2005] and [Ungerleider et al., 2002]). We studied local and long-range oscillatory brain responses by means of the electroencephalogram (EEG) in a visually guided power-grip task designed to incorporate real-world motor system requirements. This was achieved by including a continuous adjustment of the force exerted on a power grip while monitoring the visual feedback of the force output. We hypothesised that any effect of learning should be primarily reflected in increased long-range coherence as indicated by studies on the learning of bimanual coordination tasks ( [Andres et al., 1999] and [Serrien and Brown, 2003]) and visuotactile learning (Miltner et al., 1999). With regard to local oscillatory activity we expected an attenuation in the movement-related power decrease (event-related desynchronisation, ERD) (Pfurtscheller, 1977) following task practice, as visuomotor learning has been found to go along with reduced cortical activity ( [Floyer-Lea and Matthews, 2004] and [Smith et al., 1999]). Moreover, we aimed to explore whether oscillatory activity observed in preparation for a motor task ( [Babiloni et al., 2006] and [Gomez et al., 2004]) or following a motor task is also affected by learning. Effects of learning on preparatory brain responses have been previously described for the ERP (Staines et al., 2002). Oscillatory brain activity following a motor task is usually characterised by a prominent event-related synchronisation (ERS) (Pfurtscheller, 1992) over sensorimotor brain areas in the beta-frequency range, also known as beta-rebound (Parkes et al., 2006). Pre-movement changes have been reported in terms of an ERD of the sensorimotor alpha rhythm (Labyt et al., 2003). To the best of our knowledge short-term learning effects have neither been described for preparatory oscillatory brain activity nor for oscillatory brain activity following a movement.

\section{Methods}

\subsection{Participants}

Participants $(n=13)$ were recruited from the student population of the University of Surrey. Data from two participants were discarded because of poor EEG data quality. The final sample size was $n=11$ (3 male), mean age 25.8 years (range $19-34, S D=4.6$ ). One participant was ambidextrous (handedness score 53.85), all other participants were predominantly right handed with scores of 62.50 and above in the Edinburgh handedness inventory (Oldfield, 1971).

Written informed consent was obtained prior to the experiment. The study complied with the declaration of Helsinki and was approved by the University of Surrey Ethics Committee. All participants were free of known past or present mental health or neurological problems and received monetary compensation for their participation. 


\subsection{Procedure and setup}

The task consisted of trailing a target force by applying a varying force to a power grip. Continuous feedback on force output was presented on a screen placed $1 \mathrm{~m}$ in front of the participant. The forcefeedback display consisted of a vertical thermometer-like scale, with $0 \%$ representing no force, and $100 \%$ representing maximum target force (MTF), a blue vertical bar representing the force exerted when squeezing the power grip, and a red horizontal bar representing the target force, all presented on a black background. MTF refers to the maximum value the pre-specified force track could take on. Trial layout, power grip, and force track are illustrated in Fig. 1.

The maximum force level (MFL) measurable with the power-grip's force sensors was $3 \mathrm{~kg}$ or $29.42 \mathrm{~N}$. Preliminary tests indicated that this force level could be reached by healthy participants without problems. For this reason maximum voluntary contraction was not measured. Rather, the MTF was set to $2.1 \mathrm{~kg}$ or $20.6 \mathrm{~N}$ for all participants.

Each trial began with a cue presented for $500 \mathrm{~ms}$. Following the cue the screen remained blank for $846 \mathrm{~ms}$ completing the cuing period, before the force-feedback display was presented. The appearance of the force-feedback display indicated to the participant to commence the movement by squeezing the power grip (cf. Fig. $1 \mathrm{~A}-\mathrm{C}$ and Fig. 1, right-hand inset). Participants were instructed to press the power grip as hard as necessary with their right hand, or to release it, to match the tip of the vertical bar (exerted force) and the horizontal bar (target force). When matching was achieved, the horizontal bar turned green. The target force remained at the constant level of 50\% MTF for $1000 \mathrm{~ms}$, giving the participant time to reach the initial target force (in the following this will be referred to as ramp phase). Then the target force started to vary continuously along a vertical scale following a prespecified force track (cf. Fig. 1 left-hand inset) for a further $5250 \mathrm{~ms}$ (referred to as continuous change phase). Participants had to continuously adjust the exerted force in order to track the target force level. After that the screen remained blank for an average of $5500 \mathrm{~ms}$ (range 5000-6000 ms, cf. Fig. 1D), before a new cue announced the beginning of the next trial. Participants were instructed to ease their grip of the power grip as soon as the feedback display disappeared as much as possible, but not to let go. Force data were only collected during trials when the force-feedback display was shown.

The task was presented at two levels of difficulty; task difficulty was manipulated by the gain in the feedback signal associated with the exerted force. In the more difficult condition that is in the focus of the present report, gain was high: changes in the exerted force resulted in large changes in the feedback signal, making it difficult to keep the exerted force in the target force range. Behavioural pilot data suggested that performance improvements are particularly evident with high feedback gain (Sterr et al., 2006). In the easier condition (not reported ${ }^{1}$ ) similar changes in the exerted force resulted in smaller changes (factor 0.5). The cue presented before task onset indicated whether the subsequent trial would be of the high-accuracy (cue ' $\mathrm{H}$ ') or low-accuracy type (cue 'E').

Stimulus size at the display was as follows: cue $2 \times 1.3 \mathrm{~cm}$, force-feedback display $14.4 \times 9 \mathrm{~cm}$, $\mathrm{red} / \mathrm{green}$ horizontal bar (target force) $2.5 \times 1 \mathrm{~cm}$, and blue vertical bar (exerted force) $4 \mathrm{~mm}$ in width. The force applied to the power grip was sampled at about $70 \mathrm{~Hz}$ and directly projected to the screen, so that there was no discernible lag between the exerted force and feedback. Experimental control was established using Presentation Version 10.0 in combination with Matlab (for technical details see Hou et al., 2005).

A total of 90 trials for either feedback-condition were run in six blocks of 30 trials each; the present analyses focus on the first and the second and the fifth and the sixth block, respectively. Highaccuracy and low-accuracy feedback-conditions were randomised within blocks. Participants were given short brakes of about 1 min between blocks; the whole experiment lasted approximately $1 \mathrm{~h}$. 


\subsection{Electroencephalographic recordings}

EEG recordings were conducted using an electrode cap on which $64 \mathrm{Ag}-\mathrm{AgCl}$ electrodes were mounted (EASYCAP, Herrsching, Germany) according to the 10-10 system. In addition, horizontal and vertical eye movements were recorded bipolarly from two electrodes placed above and below the left eye, and two further electrodes placed at the outer canthi of the eyes. The ground electrode was placed on the forehead. Recording reference was the online calculated common average of all scalp channels. Data were recorded using the high impedance 72 channel Brain Vision Quickamp amplifier and Brain Vision Recorder software version 1.03 (Brain Products, Gilching, Germany). Sensor impedances were kept below $20 \mathrm{k} \Omega$ prior to data acquisition (cf. [Debener et al., 2007], [Ferree et al., 2001], [Gruber and Müller, 2005], [Kranczioch et al., 2007] and [Melloni et al., 2007]). Data were recorded at a sampling rate of $500 \mathrm{~Hz}$ in the DC mode.

\subsection{Data analysis}

Performance was quantified as deviation of the exerted force from the target force with a tolerance of $\pm 3.75 \% \mathrm{MVL}$. That is, whenever the exerted force was below or above the target force range, the absolute value of the force difference was calculated. Calculations were based on arbitrary units (AU), with 400 reflecting the $\mathrm{MVL}$ of $3 \mathrm{~kg}$, and $3.75 \%$ corresponding to $15 \mathrm{AU}$ or $112.5 \mathrm{~g}$. If the exerted force was within the target force range, this difference was zeroed. For each experimental block of the high-accuracy condition and participant the sum of differences or errors was then calculated across time for the 1st to the 50th, and the 165th to the 475th sampling points to obtain a measure of error amplitude. Sampling point ranges, respectively, correspond to the time ranges task onset to about $2100 \mathrm{~ms}$ (ramp phase), and about $2500 \mathrm{~ms}$ to about $7125 \mathrm{~ms}$ (continuous change phase, see Fig. 1, left-hand inset). It was furthermore calculated how often errors occurred during the ramp and continuous change phases. To this end the number of sampling points for which the exerted force was outside the target force range was counted for each trial of the high-accuracy condition. For each participant, counts were summed across the 15 trials of each block and divided by the number of trials.

EEG data analysis was performed with EEGLAB 4.51 and 5.03 (Delorme and Makeig, 2004), a freely available open source software toolbox (Swartz Center for Computational Neurosciences, La Jolla, CA; http://www.sccn.ucsd.edu/eeglab) running under Matlab (MathWorks, Inc, Natick, MA). Downsampled $(250 \mathrm{~Hz})$ and $0.5-\mathrm{Hz}$ high-pass filtered data were epoched into non-overlapping segments of $11.5 \mathrm{~s}$ duration ( -1.0 to $10.5 \mathrm{~s}$ relative to cue presentation) and screened for artefacts. Following rejection of epochs containing non-stereotyped artefacts (e.g., swallowing, cable movement, etc.) concatenated single-trial EEG data were submitted to extended infomax-independent component analysis ( [Bell and Sejnowski, 1995] and [Lee et al., 1999]). Independent components reflecting eye movements or other artefacts (e.g. line noise) were identified visually and discarded by back-projecting all but these components to the data space ( [Hine and Debener, 2007] and [Kranczioch et al., 2007]). Back-projection was performed on $0.1 \mathrm{~Hz}$ filtered, epoched data, from which all previously determined trials containing non-stereotyped artefacts had been discarded.

Time-frequency analysis was performed for all channels by convolving the single-trial data with Hanning-windowed sinusoidal wavelets (Delorme and Makeig, 2004). For each trial the mean baseline log power spectrum was subtracted from the spectral estimate to produce baselinenormalised time-frequency representations. The baseline period was set to -600 to $-200 \mathrm{~ms}$ relative to cue onset. Single-trial spectral estimates were then averaged for each subject. In order to study effects of task repetition and thus learning, trials were averaged for the first and second as well as the fifth and sixth blocks, respectively.

The phase relation of the signals at different regions was analysed by computing the phase coherence (PCOH) as described in Delorme and Makeig (2004). The PCOH measure takes values between 0 and 1, where a value of 0 represents absence of synchronisation and a value near 1 indicates perfect synchronisation. To compute $\mathrm{PCOH}$ first the length of each of the trial activity vectors are normalised to 1 , then their complex average is computed. Thereby only the information about the phase of the spectral estimate of each trial is taken into account. To reduce the number of 
possible pairings, electrodes were collapsed into 12 topographical regions of interest $(\mathrm{ROI})$ covering the whole scalp and $\mathrm{PCOH}$ was calculated between the $12 \mathrm{ROls}$. Electrode clusters belonging to each ROI are depicted in Fig. 2A. Again, trials were combined for the first two and the last two experimental blocks.

\subsection{Statistical analysis}

All statistical analyses were conducted with SPSS 12.0 and Matlab 7.0.1. To analyse effects of task practice on performance, error sums and error occurrences of the first and second, and fifth and sixth experimental blocks were, respectively, added and divided by two. In the following, for all analyses the first and second blocks will be referred to as early blocks, and the fifth and sixth block will be referred to as late blocks. Error values were statistically compared using $t$-tests for dependent measures.

Inspection of the numerical values of the time-frequency spectra averaged across electrodes and participants indicated that power changes were most prominent at $9.38 \mathrm{~Hz}\left(\sigma_{\mathrm{f}}=1.56\right), 13.13 \mathrm{~Hz}\left(\sigma_{\mathrm{f}}=\right.$ $1.64)$, and $19.69 \mathrm{~Hz}\left(\sigma_{\mathrm{f}}=1.71\right)^{2}$. Accordingly, these three frequency ranges representing the alpha $(8-12 \mathrm{~Hz})$ and lower beta frequency bands $(13-21 \mathrm{~Hz})$ were selected for further statistical analysis as well as $\mathrm{PCOH}$ analysis. The three frequency ranges will be referred to as 9,13 , and $20 \mathrm{~Hz}$ range in the following. Activity in the 13 and $20 \mathrm{~Hz}$ range was analysed separately as the time-frequency representation indicated different patterns of results for these two frequencies.

Statistical analysis of EEG data focused on five time windows relative to cue onset (cf. Fig. 2B): early preparatory (EP), late preparatory (LP), early motor (EM) reflecting the ramp phase of the movement, late motor (LM) reflecting the phase of continuous movement, and post movement (PM). Corresponding time windows in the present data set were 302-496 ms (EP), 792-1347 ms (LP), 1359-2094 ms (EM), 2507-3499 ms (LM), and 8798-9700 ms (PM). Time windows were chosen based on task characteristics and to reflect the main properties of the results of the power analysis. The differentiation between early and late preparatory and motor phases was furthermore based on previous studies that implicated functional differences between early and late preparatory activity as well as between activity during the initiation of a movement and the continuation of the movement (e.g., Rearick et al., 2001). For two of the time windows (EP in the $9-\mathrm{Hz}$ and PM in the 13- $\mathrm{Hz}$ range) task-related changes in activity were slightly shifted as evident from the time-frequency representations (cf. Fig. 4A and B). To account for the shift the EP time window was adjusted to $302-$ 779 ms for analysing activity in the 9-Hz range, and the PM time window was adjusted to 8205-8991 $\mathrm{ms}$ for analysing activity in the $13-\mathrm{Hz}$ range.

To statistically test power changes related to task practice and learning, separate repeated measures ANOVAs were run for all time-frequency windows. Electrodes were combined in 12 ROls (cf. Fig. 2A) in order to avoid a loss of statistical power (Oken and Chiappa, 1986). The 3-factorial repeatedmeasures ANOVAs comprised the region factors laterality (left, midline, and right) and caudality (frontal, fronto-central, posterior, and occipital), and the experimental factor learning. The factor learning had two levels (early and late), where the level early comprised the first and second and the level late the fifth and sixth experimental blocks. Significant interactions involving the factor learning were followed by post-hoc tests. Where necessary, Huynh-Feldt correction was applied (Huynh and Feldt, 1976); corrected $p$-values are reported with uncorrected degrees of freedom.

Statistical analysis of coherence values focused on the three frequency ranges most clearly displaying task-related power changes, i.e. the 9-, 13-, and 20- $\mathrm{Hz}$ ranges. This strategy has been successfully applied before (e.g., [Gross et al., 2004], [Kranczioch et al., 2007] and [Melloni et al., 2007]). It was based on ROI values and focused on time or learning effects. For early and late blocks $\mathrm{PCOH}$ was compared to a pre-cue baseline period of -600 to $-200 \mathrm{~ms}$. PCOH values were also directly compared between early and late runs. Non-parametric Friedman tests were run to compare $\mathrm{PCOH}$ of the baseline period and early and late blocks for all ROI pairs and time-frequency windows. When the Friedman test was significant $(p \leqslant 0.05)$ for an ROI pair, a multiple comparison test based on the Studentised range distribution and using Tukey's honestly significant difference criterion was 
run to indicate for which of the three possible combinations (early blocks-baseline, late blocksbaseline, and early blocks-late blocks) a significant difference $(p \leqslant 0.05)$ existed.

\section{Results}

\subsection{Behavioural data}

Learning was evident by an improvement of performance over time, that is, the sum of errors or error amplitude as well as error occurrence was higher in the early blocks as compared to the late blocks. For the sum of errors this difference was significant for the continuous change phase $(t(10)=4.3$, $p$ (one-tailed $)=0.001$; cf. Fig. $3 \mathrm{~B})$, but not for the earlier ramp phase $(t(10)=1.4, p$ (one-tailed $)=$ 0.095; cf. Fig. 3A). Similar results were obtained for error occurrence, where the difference was significantly different for the continuous change phase $(t(10)=4.4, p$ (one-tailed) $=0.0015)$, but not the ramp phase $(t(10)=1.0, p$ (one-tailed) $>0.2$ ).

\subsection{EEG data}

Fig. 4A and $B$ show grand mean average time-frequency plots (averaged across all scalp channels and participants), topographies for the 9-, 13-, and 20-Hz ranges for early and late blocks, and ROI pairs for which the statistical analyses indicated that coherence was significantly increased for either experimental condition, that is, early or late blocks, or the baseline period. Following cue onset an ERD was observed for the 9- and $20-\mathrm{Hz}$ ranges which was maximal over left centro-parietal electrode sites. By the end of the cuing period this ERD had largely disappeared. Following task onset the ERD returned, though much stronger and more bilaterally distributed, and was sustained until after task offset. About $1000 \mathrm{~ms}$ following task offset a prominent ERS became evident as a beta-rebound that was maximal for the 13- $\mathrm{Hz}$ range and had a fronto-central medial and occipital distribution. In the following, learning-related effects for power and coherence will be presented separately for each phase of the task, that is movement preparation, movement execution and post-movement.

\subsubsection{Movement preparation}

In the $9-\mathrm{Hz}$ range, the ANOVA for the EP time window revealed a main effect of the factor learning, indicating that the ERD was significantly increased for the late blocks (time, $F(1,10)=13.6, p=$ 0.004). No significant power differences were found for the LP time window.

In the LP time window coherence was increased for the early as compared to the late blocks of the experimental task over a wide range of regions for the $13-$ and $20-\mathrm{Hz}$ frequency ranges, particularly including frontal and parietal ROls (see Fig. 4C).

\subsubsection{Movement execution}

No significant differences were apparent for the EM time window. For the LM time window a significant learning $\times$ caudality interaction $(F(1,10)=3.216, p=0.037)$ was found for the $20-\mathrm{Hz}$ range, that reflected a larger ERD for early than for late blocks. However, as indicated by post-hoc $t$-tests this effect was restricted to the fronto-central ROIs $(t(10)=-2.01, p$ (one-tailed) $=0.036$ ).

The EM time range showed relative increases in coherence for early as well as late blocks (see Fig. 4C). For all three frequency ranges studied, for early blocks coherence was particularly increased between fronto-central ROIs ipsilateral to the moving hand and contralateral parieto-occipital ROls. At the same time, particularly for the $13-\mathrm{Hz}$ range, coherence was increased for late blocks between contralateral and medial fronto-central ROIs and the ipsilateral parietal region. In the LM time range a hemispheric shift of coherence patterns was observed in that now, particularly for the $13-$ and $20-\mathrm{Hz}$ range, increases in coherence were observed for late blocks between ipsilateral and medial frontal, parietal, and occipital ROls. Predominantly for the $20-\mathrm{Hz}$ range this was accompanied by an increase in coherence for early blocks between contralateral frontal and parietal ROls and all three occipital ROls (all results cf. Fig. 4C). 


\subsubsection{Post-movement}

For the $9-\mathrm{Hz}$ range $\mathrm{PM}$ time window a main effect for the factor learning $(F(1,10)=5.43, p=0.042)$ and an interaction of the factors learning $\times$ caudality $(F(1,10)=3.3, p=0.046)$ indicated that the ERS was larger for the early as compared to the late runs, in particular at frontal and occipital ROls. Similarly to the $9-\mathrm{Hz}$ results, for the $13-\mathrm{Hz}$ frequency range the ERS was stronger for the early blocks (main effect learning, $F(1,10)=5.96, p=0.035$ ) in time window PM. This effect was most pronounced at frontal and occipital ROls (learning $\times$ caudality, $F(1,10)=4.224, p=0.013$ ).

Increased coherence between ipsi- and contralateral and medial ROls was evident for the late blocks mostly for the $13-\mathrm{Hz}$ range. Coherence increases for the early runs predominated between frontal and occipital ROls, in particular for the $9-\mathrm{Hz}$ range (all results cf. Fig. 4C).

\section{Discussion}

The present study investigated learning-related changes in oscillatory brain activity in a visually guided, continuous force-feedback task that afforded continuous monitoring and adjustment of motor output. Learning was confirmed by significant improvements in the accuracy of motor output between early and late experimental blocks. We focused on two measures of oscillatory brain activity, namely, power and coherence. Long-range coherence has been described in a variety of EEG and magnetoencephalographic (MEG) studies, including perception ( [Rodriguez et al., 1999] and [Srinivasan et al., 1999]), attention and visual awareness ( [Gross et al., 2004] and [Kranczioch et al., 2007]), memory and learning ( [Gruber and Müller, 2005], [Miltner et al., 1999] and [Sarnthein et al., 1998]) and motor tasks ( [Classen et al., 1998], [Pollok et al., 2005] and [Serrien and Brown, 2003]). It is generally assumed that coherent activity reflects the interaction between brain areas involved in a given task, and that the mode of reciprocal interactions is the phase synchronisation between the participating neuronal groups ( [Engel et al., 2001], [Singer, 1999] and [Varela et al., 2001]). Power changes on the other hand are assumed to reflect alterations in the activity of local interactions between main neurons and interneurons. For the alpha and lower beta frequency bands a reduction in power relative to a reference interval is interpreted as a correlate of activated cortical areas involved in processing sensory or cognitive information or production of motor behaviour ( [Pfurtscheller, 1992] and [Pfurtscheller and Lopes da Silva, 1999]).

In general, in accordance to previous research we found that motor learning was associated with both increases and decreases in neuronal activity. However, in contrast to the present study previous research focused on bimanual coordination tasks ( [Andres et al., 1999] and [Serrien and Brown, 2003]), or used fMRI (Floyer-Lea and Matthews, 2004). Also, none of these studies took into consideration brain activity related to motor preparation and the post-movement phase. Here we provide evidence that improved task performance in a visually guided unimanual power-grip task is associated with changes in both EEG power and coherence at different times. Importantly, we observed learning-related effects during the execution of the movement, the preparatory phase, and the post-movement phase. Learning-related changes differed between these phases but also within each phase, suggesting a considerable dynamic in the underlying brain activity. In the following we will discuss the results separately for each phase of the task and close with a paragraph integrating the results.

\subsection{Movement preparation}

The early time range of the movement preparation phase (EP) was characterised by an ERD in the 9$\mathrm{Hz}$ (alpha) and $20-\mathrm{Hz}$ (beta) frequency ranges. Only for the alpha activity we observed an increase in ERD with increasing task practice. Pre-movement $\mu$ ERD $(8-12 \mathrm{~Hz})$ has been related to the unconscious preparation of voluntary movement (Labyt et al., 2003). This preparation is assumed to correspond to a relatively unspecific pre-activation or priming of neurons in motor areas ( [Gomez et al., 2004] and [Pfurtscheller and Lopes da Silva, 1999]). The present results suggest that with increasing task practice larger neuronal networks were activated in preparation for the task or that activity within existing networks is amplified to subserve improved performance. In support of this 
suggestion linking pre-task ERD and motor task performance, good or expert performance in nonmotor tasks (e.g., [Ergenoglu et al., 2004], [Hanslmayr et al., 2005] and [Kranczioch et al., 2007]) and in the aiming period of a target shooting task (Haufler et al., 2000) has been variously related to reduced pre-task alpha power. For the late preparatory time range (LP) we found a widespread reduction in coherence with task practice in the beta frequency range, particularly involving frontal and parietal ROls. Interestingly, in a study focusing on long-term aspects of motor learning it was found that during an aiming period, expert marksmen displayed less alpha and beta coherence than skilled shooters, particularly in the left hemisphere (Deeny et al., 2003). This was interpreted as reflecting that experts engage in less cortico-cortical communication. Babiloni et al. (2006) report on contralateral centro-parietal functional coupling during the anticipation of motor events, reflecting functional coordination of these areas. However, their study was not looking for learning effects, and was restricted to electrodes overlying central and parietal cortical areas. Moreover, they argue themselves that anticipatory sensorimotor interaction is likely not be limited to these, but to include multiple frontal, parietal, and sub-cortical structures that among others have been described to subserve top-down attentional or anticipatory influences ( [Babiloni et al., 2006], [Giesbrecht et al., 2003] and [Pessoa et al., 2003]). Our results support this proposal as they demonstrate that the preparation for a motor event does involve more than centro-parietal areas, a view also supported by recent ERP and source modelling studies ( [Mathews et al., 2006] and [Praamstra et al., 2005]). Moreover, recent research has related fronto-parietal beta coherence to the activation of an attention network ( [Gross et al., 2004] and [Kranczioch et al., 2007]). Thus, it can be speculated that shortterm motor learning might be associated with a reduction of top-down attentional influences, at least in the preparatory phase of the movement.

In sum, our results suggest that the repeated performance of a complex motor task affects the preparation for this task twofold: On the one hand in terms of an increase in local motor area preactivation or priming, and on the other hand in terms of a decrease in general coupling between brain areas. Whereas the first mechanism might reflect a better preparation of brain areas directly involved in executing the upcoming movement, it might be speculated that the latter indicates that less topdown attentional control and less communication between brain areas is required in preparation for the task.

\subsection{Movement execution}

At the beginning of the movement execution phase (EM) coupling between ipsilateral fronto-central and contralateral parieto-occipital ROls was increased at alpha ( $9 \mathrm{~Hz}$ range) and beta (13 and $20 \mathrm{~Hz}$ ranges) frequencies when the task was new. In contrast, after practice coupling was larger between contralateral fronto-central and ipsilateral parietal electrode clusters, though only at the lower beta frequency range. In the late movement execution phase (LM), fronto-central $20-\mathrm{Hz}$ range beta ERD was attenuated following task practice, so was coherence between contralateral frontal and parietal, and all occipital ROls. On the other hand, beta coherence (13 and $20 \mathrm{~Hz}$ ranges) amongst ipsilateral frontal, parietal, and occipital ROls increased with task practice.

The fronto-centrally focused reduction in ERD with increasing task practice indicates increasingly less motor-related activation during task execution, particularly in pre-central cortical areas. Alternatively, it might reflect an increase in the synchronisation of neuronal activation. Even though this possibility cannot be fully ruled out, visual inspection of inter-trial coherence values indicated no difference between early and late blocks, which makes it highly unlikely. The conclusion that the reduction in ERD reflects less motor-related activation is also in accordance to a previous fMRI study that showed that visuomotor short-term learning is associated with reduced activation in primary motor cortex and prefrontal brain areas (Floyer-Lea and Matthews, 2004). That changes in ERD can be paralleled by changes in fMRI BOLD responses have been suggested previously for upper alpha ERD (Jäncke et al., 2006). Also in support of a learning-related decrease in cortical activity is a study by Slobounov et al. (2007) in which an increase in central alpha activity was found following several weeks of practicing a finger force production task. In sum, the reduction in ERD might be a correlate of the movement execution to become less effortful and less attentional demanding. 
The finding of coherence patterns that include electrode clusters over frontal and fronto-central, parietal and occipital brain regions is also not surprising, given that it has been previously reported that visuomotor integration - in contrast to visual input and motor output not requiring integration - is characterised by increased coherence between these regions, in particular between central, right (ipsilateral) parietal, and occipital electrodes (Classen et al., 1998). This interregional coherent activity is presumably causally related to a cooperativity between regions involved in visuomotor integration (Classen et al., 1998). Following somatosensory associative learning Miltner et al. (1999) further observed enhanced coherence between electrodes overlying occipital and contralateral somatosensory cortex regions. They argue that the increase in coherence might reflect the binding of the brain areas that must communicate with one another to promote learning.

The results of the present study indicate that performance improvements, and thus, motor learning can be associated with different coherence patterns for the different sections of the studied motor task. In particular, performance in the phase of movement initiation seems to benefit from an increase in coherence between contralateral frontal/fronto-central and ipsilateral parieto-occipital brain regions, and a parallel decrease in coherence between ipsilateral fronto-central and contra-lateral parietooccipital brain regions, potentially reflecting the strengthening of an existing (contralateral) neuronal network. Following movement initiation the improvement of the complex, continuous movement seems to rely primarily on activity within an ipsilateral-medial network, though initially the movement seems to rely stronger on contralateral aspects of the network. Interestingly, ipsilateral centro-parietal brain regions have been related to sensorimotor integration demands during the precise control of grip forces (Ehrsson et al., 2001). FMRI grip-force studies particularly interested in motor learning ( [Floyer-Lea and Matthews, 2004] and [Floyer-Lea and Matthews, 2005]) did however not find evidence for a particular relevance of ipsilateral brain structures for improved task performance. This might indicate that improvements are not so much related to an increase in ipsilateral brain activity but to an increase in functional connectivity between contralateral and ipsilateral brain areas.

In sum, the present results support the idea that the learning of complex motor behaviours is associated with the weakening of some and strengthening of other functional connections, as has been previously noted by [Serrien and Brown, 2003] and [Andres et al., 1999] for bimanual coordination tasks.

\subsection{Post-movement}

In the post-movement phase (PM), an ERS was evident mostly for the lower beta (13 Hz range), but also for the alpha frequency range. This ERS was most pronounced over fronto-central medial and occipital ROls, and larger when the task had not yet been practiced. Coherence patterns differed between alpha and beta frequencies: alpha coherence was increased for the early experimental block between frontal-medial, occipital, and ipsilateral central and parietal ROls; while for the lower beta frequency coherence was increased following task practice between ipsi- and contralateral as well as medial ROls.

The spatial resolution of the EEG is not sufficient to allow for firm conclusions about the cortical origin of the effects observed here. However, the topographies relating to the ERS are suggestive of an origin in visual as well as medial frontal areas, probably cingulate and supplementary motor areas (SMA) (Labyt et al., 2003). This topography together with a lack of contralateral ERS can be regarded as rather unusual. Contralateral ERS is widely seen as an indicator of deactivated, 'idling' motor cortex and interpreted as a signature of a post-movement resetting or inhibitory control of cortical areas involved in movement planning and execution ( [Hummel et al., 2002] and [Pfurtscheller et al., 1996]), though Bender et al. (2004) propose that it is likely to be more than an 'idling' state of the whole motor system. Another functional interpretation of beta ERS is to reflect the sensory processing of proprioceptive, somesthetic, or visual reafferent inputs (Labyt et al., 2003). A third though quite speculative interpretation of the front-central medial ERS is that it reflects performance evaluation after the task in order to improve task performance. With regard to occipital alpha activity, it is considered as the 'idling' rhythm of the visual system (Kuhlman, 1978).

ERS decreased with increasing task practice, indicating that it might be related to improvements in task performance. Similar to the ERD results it suggests that task practice went along with less 
cortical activation (and hence less deactivation/inhibition), and is in accordance with fMRI data that indicate a decrease in activation in fronto-medial cortical areas with improving visuomotor performance (Floyer-Lea and Matthews, 2004). Among other functions, the SMA seems to be involved in movement control ( [Cunnington et al., 2002], [Floyer-Lea and Matthews, 2004], [Stephan et al., 1995] and [Vaillancourt et al., 2003]) which would support the idea of ERS reflecting a reset of cortical activity in brain regions involved in a motor task as expressed in the 'idling' hypothesis (Pfurtscheller et al., 1996). On the other hand, the SMA receives proprioceptive input from sensory afferents stimulated by passive movements (Radovanovic et al., 2002), supporting the reafferent input hypothesis of ERS ( [Cassim et al., 2001] and [Labyt et al., 2003]). Another medial frontal structure, the anterior cingulate cortex (ACC), has been suggested to have a fundamental role in relating actions to their consequences (Rushworth et al., 2004). It can be speculated that in the early blocks performance evaluation and relating actions to consequences is more important than in the late blocks. Accordingly, early blocks would be associated with higher activity in frontal-medial structures such as the ACC. The sensitivity of occipital ERS to task practice might indicate that participants became adapted to the visual stimulation, resulting in less cortical activation during the task and accordingly less inhibition/deactivation after task offset.

A possible explanation why nearly no ERS was observed over primary sensorimotor areas is that even though the motor task was demanding, it did not involve much movements of great extent, but subtle modulations of the muscular tone in the finger extensors. In addition, though participants were instructed to loosen their hold of the power grip it remained in their hand when the task was finished and throughout the baseline period, resulting in a continuous sensorimotor input rather than an 'idling' state of sensorimotor cortex.

Significant coupling between the two hemispheres during the post-movement period has neither been observed for central beta nor mu rhythms ( [Andrew and Pfurtscheller, 1996], [Andrew and Pfurtscheller, 1999], [Kilner et al., 2003] and [Storm Van Leeuwen et al., 1978]). This has been taken as evidence for the independence of the neural circuits responsible for the generation of these rhythms in the left and right hemispheric hand motor regions ( [Andrew and Pfurtscheller, 1999] and [Kilner et al., 2003]). The present results are partially in line with these observations, as learningrelated differences in coupling were not observed between ipsi- and contralateral fronto-central ROls. However, distinct patterns of long-range coherence were observed for the early and the late stages of visuomotor learning, providing evidence for post-movement cortico-cortical interaction. Though highly speculative at this point, post-movement alpha coherence patterns during the early experimental blocks might be a correlate of the synchronous inhibition of frontal and occipital brain areas also reflected in the post-movement power increase. On the other hand, lower beta coherence patterns found for the late experimental blocks may reflect the re-establishing of interhemispheric communication following primarily contralateral (early phase movement execution) or ipsilateral (late phase movement execution) coupling.

\subsection{Oscillatory activity as an indicator of the dynamics of short-term motor learning}

The present study demonstrates the complex brain dynamics underlying the performance of a multifaceted motor adaptation task. We could replicate previous findings of a learning-related reduction of motor-related local activation during movement execution, supporting the idea that regional practice-related changes might reflect the development of task specific neurocognitive strategies (Smith et al., 1999). A new finding is that motor-related activation during the preparation for the visuomotor task increased rather than decreased with learning. Differences in pre-movement ERD were focused in the alpha band (mu), whereas during the movement learning effects were largest in the beta band. This suggests that partially different populations of motor neurons were activated during motor preparation and motor execution (Pfurtscheller and Lopes da Silva, 1999). Alternatively, the results might reflect that practice leads to an increase in the general readiness or pre-activation of sensorimotor neurons (Pfurtscheller et al., 1998), resulting in relatively less activation of the same population during movement execution.

The analysis of long-range coherence revealed several novel findings that taken together indicate that the observed behavioural improvements were associated with dynamic changes in cortico-cortical coupling. Changes were found both across the different phases of the visuomotor task as well as with task practice and thus learning. Coherence patterns varied, but almost without exception included frontal or fronto-central, parietal and occipital areas, emphasising the complexity of the network 
realising visuomotor integration. This is in accordance with a model of motor learning postulating that the early stage of motor skill acquisition both for motor adaptation as well as motor sequence learning relies crucially on the dynamic interactions between subcortical regions and motor, prefrontal, parietal and cortical limbic areas (Doyon and Benali, 2005). Depending on the phase of the task corticocortical coupling may reflect top-down attentional control, the strengthening or weakening of brain networks to accomplish the different aspects of the visuomotor integration task, and finally the inhibition of activity of brain areas that are part of the network or a post-movement re-establishing of interhemispheric communication.

\section{Conclusions}

The picture emerging from the present study is that changes in cortico-cortical coupling as well as in local oscillatory activity might promote visuomotor learning. Local oscillatory activity indicates that behavioural improvements go along with increases in preparatory and decreases in execution-related sensorimotor activity as well as with decreases in the post-movement "idling" state of medial-frontal and occipital brain areas. Coherence data suggest that motor learning might be associated with less preparatory attentional processes and an emphasis on joint activity of primarily contralateral parts of the visuomotor network during movement initiation as opposed to a focus on primarily ipsilateral brain areas during the continuous movement phase. Results furthermore suggest that following the movement inhibitory processes as well as re-establishing interhemispheric connections might be of importance. Whether these practice-related changes of local activity and long-range coherence are characteristic for short-term visuomotor learning or can also be observed over longer periods of time remains to be elucidated by future studies.

\section{Acknowledgement}

We are grateful to Phil Dean for his contribution to data recordings.

\section{REFERENCES}

Andres FG, Mima T, Schulman AE, Dichgans J, Hallett M, Gerloff C. Functional coupling of human cortical sensorimotor areas during bimanual skill acquisition. Brain 1999;122(Pt. 5):855-70.

Andrew C, Pfurtscheller G. Event-related coherence as a tool for studying dynamic interaction of brain regions. Electroencephalogr Clin Neurophysiol 1996;98(2):144-8.

Andrew C, Pfurtscheller G. Lack of bilateral coherence of post-movement central beta oscillations in the human electroencephalogram. Neurosci Lett 1999;273(2):89-92.

Aoki F, Fetz EE, Shupe L, Lettich E, Ojemann GA. Increased gammarange activity in human sensorimotor cortex during performance of visuomotor tasks. Clin Neurophysiol 1999;110(3):52437.

Babiloni C, Brancucci A, Vecchio F, Arendt-Nielsen L, Chen AC, Rossini PM. Anticipation of somatosensory and motor events increases centroparietal functional coupling: an EEG coherence study. Clin Neurophysiol 2006;117(5):1000-8.

Baker JT, Donoghue JP, Sanes JN. Gaze direction modulates finger movement activation patterns in human cerebral cortex. J Neurosci 1999;19(22):10044-52. 
Bell AJ, Sejnowski TJ. An information-maximization approach to blind separation and blind deconvolution. Neural Comput

1995;7(6):1129-59.

Bender S, Oelkers-Ax R, Resch F, Weisbrod M. Motor processing after movement execution as revealed by evoked and induced activity. Brain Res Cogn Brain Res 2004;21(1):49-58.

Cassim F, Monaca C, Szurhaj W, Bourriez JL, Defebvre L, Derambure P, et al. Does post-movement beta synchronization reflect an idling motor

cortex? Neuroreport 2001;12(17):3859-63.

Classen J, Gerloff C, Honda M, Hallett M. Integrative visuomotor behavior is associated with interregionally coherent oscillations in the human brain. J Neurophysiol 1998;79(3):1567-73.

Cunnington R, Windischberger C, Deecke L, Moser E. The preparation and execution of self-initiated and externally-triggered movement: a study of event-related fMRI. Neuroimage 2002;15(2):37385.

Debener S, Strobel A, Sorger B, Peters J, Kranczioch C, Engel AK, et al. Improved quality of auditory event-related potentials recorded simultaneously with 3-T fMRI: removal of the ballistocardiogram artefact. Neuroimage 2007;34(2):587-97.

Deeny SP, Hillman $\mathrm{CH}$, Janelle CM, Hatfield BD. Cortico-cortical communication and superior performance in skilled marksmen: an EEG coherence analysis. J Sport Exercise Psychol 2003;25:188-204.

Delorme A, Makeig S. EEGLAB: an open source toolbox for analysis of single-trial EEG dynamics including independent component analysis. J Neurosci Methods 2004;134(1):9-21.

Doyon J, Benali H. Reorganization and plasticity in the adult brain during learning of motor skills. Curr Opin Neurobiol 2005;15(2):161-7.

Ehrsson $\mathrm{HH}$, Fagergren $\mathrm{E}$, Forssberg $\mathrm{H}$. Differential fronto-parietal activation depending on force used in a precision grip task: an Fmri study. J Neurophysiol 2001;85(6):2613-23.

Engel AK, Fries P, Singer W. Dynamic predictions: oscillations and synchrony in top-down processing. Nat Rev Neurosci

$2001 ; 2(10): 704-16$.

Ergenoglu T, Demiralp T, Bayraktaroglu Z, Ergen M, Beydagi H, Uresin Y. Alpha rhythm of the EEG modulates visual detection performance in humans. Brain Res Cogn Brain Res 2004;20(3):37683.

Ferree TC, Luu P, Russell GS, Tucker DM. Scalp electrode impedance, infection risk, and EEG data quality. Clin Neurophysiol

2001;112(3):536-44. 
Floyer-Lea A, Matthews PM. Changing brain networks for visuomotor control with increased movement automaticity. J Neurophysiol 2004;92(4):2405-12.

Floyer-Lea A, Matthews PM. Distinguishable brain activation networks for short- and long-term motor skill learning. J Neurophysiol 2005;94(1):512-8.

Giesbrecht B, Woldorff MG, Song AW, Mangun GR. Neural mechanisms of top-down control during spatial and feature attention. Neuroimage 2003;19(3):496-512.

Gomez CM, Vaquero E, Lopez-Mendoza D, Gonzalez-Rosa J, Vazquez- Marrufo M. Reduction of EEG power during expectancy periods in humans. Acta Neurobiol Exp (Wars) 2004;64(2):143-51.

Gross J, Schmitz F, Schnitzler I, Kessler K, Shapiro K, Hommel B, et al. Modulation of long-range neural synchrony reflects temporal limitations of visual attention in humans. Proc Natl Acad Sci USA 2004;101(35):13050-5.

Gruber T, Mu" Iler MM. Oscillatory brain activity dissociates between associative stimulus content in a repetition priming task in the human EEG. Cereb Cortex 2005;15(1):109-16.

Halder P, Sterr A, Brem S, Bucher K, Kollias S, Brandeis D. Electrophysiological evidence for cortical plasticity with movement repetition. Eur J Neurosci 2005;21(8):2271-7.

HansImayr S, Klimesch W, Sauseng P, Gruber W, Doppelmayr M, Freunberger R, et al. Visual discrimination performance is related to decreased alpha amplitude but increased phase locking. Neurosci Lett 2005;375(1):64-8.

Haufler AJ, Spalding TW, Santa Maria DL, Hatfield BD. Neuro-cognitive activity during a self-paced visuospatial task: comparative EEG profiles in marksmen and novice shooters. Biol Psychol 2000;53(2-3):131-60.

Hine J, Debener S. Late auditory evoked potentials asymmetry revisited. Clin Neurophysiol 2007;118(6):1274-85.

Hou W, Shen S, Sterr A. An MRI compatible visual force-feedback system for the study of force control mechanics. In: 27th annual international conference of the ieee engineering in medicine and biology society (EMBC05), Shanghai, China; 2005.

Hummel F, Andres F, Altenmuller E, Dichgans J, Gerloff C. Inhibitory control of acquired motor programmes in the human brain. Brain 2002;125(Pt. 2):404-20.

Huynh H, Feldt LS. Estimation of the box correction for degrees of freedom from sample data in randomized block and splitplot designs. J Educ Stat 1976;1:69-82.

Ja"ncke L, Lutz K, Koeneke S. Converging evidence of ERD/ERS and BOLD responses in motor control research. Prog Brain Res 2006;159:261-71. 
Kilner JM, Salenius S, Baker SN, Jackson A, Hari R, Lemon RN. Taskdependent modulations of cortical oscillatory activity in human subjects during a bimanual precision grip task. Neuroimage 2003;18(1):67-73.

Kranczioch C, Debener S, Maye A, Engel AK. Temporal dynamics of access to consciousness in the attentional blink. Neuroimage 2007;37(3):947-55.

Kuhlman W. Functional topography of the human mu rhythm. Electroencephalogr Clin Neurophysiol 1978;44:83-93.

Labyt E, Szurhaj W, Bourriez JL, Cassim F, Defebvre L, Destee A, et al. Changes in oscillatory cortical activity related to a visuomotor task in young and elderly healthy subjects. Clin Neurophysiol 2003;114(6):1153-66.

Lee D. Coherent oscillations in neuronal activity of the supplementary motor area during a visuomotor task. J Neurosci

2003;23(17):6798-809.

Lee TW, Girolami M, Sejnowski TJ. Independent component analysis using an extended infomax algorithm for mixed subgaussian and supergaussian sources. Neural Comput 1999;11(2):417-41.

Mathews S, Ainsley Dean PJ, Sterr A. EEG dipole analysis of motorpriming foreperiod activity reveals separate sources for motor and spatial attention components. Clin Neurophysiol 2006;117(12):2675-83.

Melloni L, Molina C, Pena M, Torres D, Singer W, Rodriguez E. Synchronization of neural activity across cortical areas correlates with conscious perception. J Neurosci 2007;27(11):2858-65.

Miltner WH, Braun C, Arnold M, Witte H, Taub E. Coherence of gamma-band EEG activity as a basis for associative learning. Nature 1999;397(6718):434-6.

Ohara S, Ikeda A, Kunieda T, Yazawa S, Baba K, Nagamine T, et al. Movement-related change of electrocorticographic activity in human supplementary motor area proper. Brain 2000;123(Pt. 6):1203-15.

Oken BS, Chiappa KH. Statistical issues concerning computerized analysis of brainwave topography. Ann Neurol 1986;19(5):493-7.

Oldfield RC. The assessment and analysis of handedness: the Edinburgh inventory. Neuropsychologia 1971;9(1):97-113.

Parkes LM, Bastiaansen MC, Norris DG. Combining EEG and fMRI to investigate the post-movement beta rebound. Neuroimage 2006;29(3):685-96. 
Pessoa L, Kastner S, Ungerleider LG. Neuroimaging studies of attention: from modulation of sensory processing to top-down control. J Neurosci 2003;23(10):3990-8.

Pfurtscheller G. Graphical display and statistical evaluation of eventrelated desynchronization (ERD). Electroencephalogr Clin Neurophysiol 1977;43:757-60.

Pfurtscheller G. Event-related synchronization (ERS): an electrophysiological correlate of cortical areas at rest. Electroencephalogr Clin Neurophysiol 1992;83:62-9.

Pfurtscheller G, Lopes da Silva FH. Event-related EEG/MEG synchronization and desynchronization: basic principles. Clin Neurophysiol 1999;110(11):1842-57.

Pfurtscheller G, Stancak Jr A, Neuper C. Post-movement beta synchronization. A correlate of an idling motor area? Electroencephalogr Clin Neurophysiol 1996;98(4):281-93.

Pfurtscheller G, Zalaudeck K, Neuper C. Event-related beta synchronization after wrist, finger and thumb movement. Electroencephalogr Clin Neurophysiol 1998;109:154-60.

Pollok B, Sudmeyer M, Gross J, Schnitzler A. The oscillatory network of simple repetitive bimanual movements. Brain Res Cogn Brain Res 2005;25(1):300-11.

Praamstra P, Boutsen L, Humphreys GW. Frontoparietal control of spatial attention and motor intention in human EEG. J Neurophysiol 2005;94(1):764-74.

Radovanovic S, Korotkov A, Ljubisavljevic M, Lyskov E, Thunberg J, Kataeva G, et al. Comparison of brain activity during different types of proprioceptive inputs: a positron emission tomography study. Exp Brain Res 2002;143(3):276-85.

Rearick MP, Johnston JA, Slobounov SM. Feedback-dependent modulation of isometric force control: an EEG study in visuomotor integration. Brain Res Cogn Brain Res 2001;12(1):117-30.

Rodriguez E, George N, Lachaux JP, Martinerie J, Renault B, Varela FJ. Perception's shadow: longdistance synchronization of human brain activity. Nature 1999;397(6718):430-3.

Roelfsema PR, Engel AK, Konig P, Singer W. Visuomotor integration is associated with zero time-lag synchronization among cortical areas. Nature 1997;385(6612):157-61.

Rushworth MF, Walton ME, Kennerley SW, Bannerman DM. Action sets and decisions in the medial frontal cortex. Trends Cogn Sci 2004;8(9):410-7.

Sarnthein J, Petsche H, Rappelsberger P, Shaw GL, von Stein A. Synchronization between prefrontal and posterior association cortex during human working memory. Proc Natl Acad Sci USA 1998;95(12):7092-6.

Serrien DJ, Brown P. The integration of cortical and behavioural dynamics during initial learning of a motor task. Eur J Neurosci 
2003;17(5):1098-104.

Singer W. Neuronal synchrony: a versatile code for the definition of relations? Neuron 1999;24(1):49_ 65, [111-25].

Slobounov S, Ray W, Cao C, Chiang H. Modulation of cortical activity as a result of task-specific practice. Neurosci Lett

2007;421(2):126-31.

Smith ME, McEvoy LK, Gevins A. Neurophysiological indices of strategy development and skill acquisition. Brain Res Cogn Brain Res 1999;7(3):389-404.

Srinivasan R, Russell DP, Edelman GM, Tononi G. Increased synchronization of neuromagnetic responses during conscious perception. J Neurosci 1999;19(13):5435-48.

Staines WR, Padilla M, Knight RT. Frontal-parietal event-related potential changes associated with practising a novel visuomotor task. Brain Res Cogn Brain Res 2002;13(2):195-202.

Stephan KM, Fink GR, Passingham RE, Silbersweig D, Ceballos-Baumann AO, Frith CD, et al. Functional anatomy of the mental representation of upper extremity movements in healthy subjects. J Neurophysiol 1995;73(1):373-86.

Sterr A, Shen S, Hou W, Gao G, Szameitat A. Neural correlates of forcecontrol: evidence obtained in a visually guided power-grip task. In HBM 2006, Florence, Italy; 2006.

Storm Van Leeuwen W, Wieneke G, Spoelstra P, Versteeg H. Lack of bilateral coherence of mu rhythm. Electroencephalogr Clin Neurophysiol 1978;44(2):140-6.

Ungerleider LG, Doyon J, Karni A. Imaging brain plasticity during motor skill learning. Neurobiol Learn Mem 2002;78(3):553-64.

Vaillancourt DE, Thulborn KR, Corcos DM. Neural basis for the processes that underlie visually guided and internally guided force control in humans. J Neurophysiol 2003;90(5):3330-40.

Varela F, Lachaux JP, Rodriguez E, Martinerie J. The brainweb: phase synchronization and largescale integration. Nat Rev Neurosci 2001;2(4):229-39. 